\title{
Uncertainty restrictions of transfer click-torque wrenches
}

\author{
Andreas Brüge \\ Physikalisch-Technische Bundesanstalt (PTB), Bundesallee 100, 38116 Braunschweig, Germany
}

\section{ABSTRACT}

For different types of click-torque wrenches, measurement uncertainty contributions of typical calibration conditions were investigated. Procedures which differ from those given in ISO 6789 were suggested for transfer measurements at calibration facilities for click-torque wrenches in order to improve their reproducibility. Examinations were carried out for influences like lever length, rise time, temperature and humidity.

\section{Section: RESEARCH PAPER \\ Keywords: Torque; click-torque wrench; ISO 6789; transfer}

Citation: Andreas Brüge, Uncertainty restrictions of transfer click-torque wrenches, Acta IMEKO, vol. 4, no. 2, article 5, June 2015, identifier: IMEKO-ACTA-04 (2015)-02-05

Editor: Paolo Carbone, University of Perugia, Italy

Received April 21, 2015; In final form June 8, 2015; Published June 2015

Copyright: (C) 2015 IMEKO. This is an open-access article distributed under the terms of the Creative Commons Attribution 3.0 License, which permits unrestricted use, distribution, and reproduction in any medium, provided the original author and source are credited

Corresponding author: Andreas Brüge, e-mail: Andreas.Bruege@ptb.de

\section{INTRODUCTION}

Click-torque wrenches (CTWs) are setting torque tools of the type II according to ISO 6789 [1], which are wrenches with a release mechanism to limit the transferable torque. In particular this definition excludes screwdrivers or wrenches with flexion bars. They are to be calibrated according to ISO 6789 , where conformity limits are required which amount to \pm 4 $\%$ and $\pm 6 \%$ respectively, for the relative deviation of the releasing value. Nevertheless, CTWs intended as transfer standards for the traceability of the calibration facilities concerned should comply with much stricter requirements.

They should correspond to the best measurement capabilities (bmcs) of laboratories accredited for CTW calibration in the German accreditation body (DAkkS). These bmcs cover the range from $0.2 \%$ to $1 \%$ (Figure 1 ).

A key comparison of the Deutscher Kalibrierdienst (DKD) based on procedures according to the ISO 6789 using CTWs demonstrated the insufficiency of the combination of the CTWs with these procedures for transfer purposes. Therefore, nowadays only indicating transfer torque wrenches can deliver the traceability of the static torque calibration in the concerning facilities according to the bmc values of calibration laboratories. But specific dynamic requests to the facilities destined for CTW calibration cannot be verified by indicating torque wrenches. bmc of click-torque wrench calibration

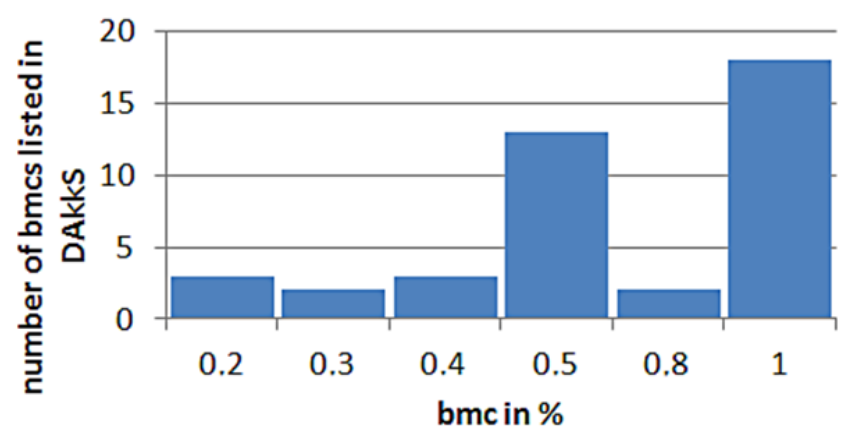

Figure 1. Distribution of listed bmcs in the DAkkS for click-torque wrench calibration according to ISO 6789.

Therefore the use of transfer CTWs is necessary particularly during assessments to complement the traceability of laboratories working in the field of ISO 6789.

In order to submit proposals for the selection of suitable types of CTWs and for measurement procedures which help overcome known restrictions of the available types, this work surveys important sources of measurement uncertainty of different types of CTWs. 


\section{CHARACTERISATION OF CTWS}

\subsection{Calibration facility}

The measurements for this paper were performed at the 2$\mathrm{kN} \cdot \mathrm{m}$ calibration facility of the Physikalisch-Technische Bundesanstalt (PTB) in Braunschweig.

This facility generates torque loads continuously using an electric motor and a gearbox. The detection of the load was conducted with Raute reference torque transducers of type TT1 and an HBM DMCplus transient recorder.

Fitted with a DV55 carrier frequency amplifier module, this recorder is able to detect releasing signals (Figure 2) under the following conditions:
carrier frequency
resolution
$4.8 \mathrm{kHz}$
sampling rate
(24 ... 15) bit
(150 ... 9600) Hz.

Altogether, the expanded relative contribution of the reference measuring chain results in approximately $8 \cdot 10^{-4}$.

\subsection{Measurements}

Calibrations of CTWs according to ISO 6789 are mainly meant to achieve the deviation of the actual release torque $A$ from the value which is set at the CTW. For this purpose, repeated releases are to be performed five times each at $20 \%$, $60 \%$ and $100 \%$ of the nominal torque. Hence inherent properties of the CTW, like running-in and repeatability, should be part of the result.

In contrast the intention of the measurements in this work is to figure out the CTW's coefficients of disturbing quantities and other contributions to their measurement uncertainty.

Furthermore, procedures are questioned in order to minimize the impact of disturbing quantities and to maximize the reproducibility during a transfer measurement in a calibration laboratory. These procedures are discussed later in this paper.

In the following, brief descriptions of the measurement procedures used for determining considerable influences on the calibration of CTWs are given.

\subsection{Release torque $\boldsymbol{A}$}

The calibration facility performs a constantly increasing load until the CTW releases and the measured torque falls sharply. The peak value $A$ of the recorded signal of the reference measuring chain is calculated, taking into account the relative noises of the release curve by detecting the span $b_{\mathrm{A}}$ (Figure 3), which is a specific value of the CTWs between $2 \cdot 10^{-5}$ and $2 \cdot 10^{-4}$ and of the reference measurement chain's zero signal (typically $3.5 \cdot 10^{-5}$ ).

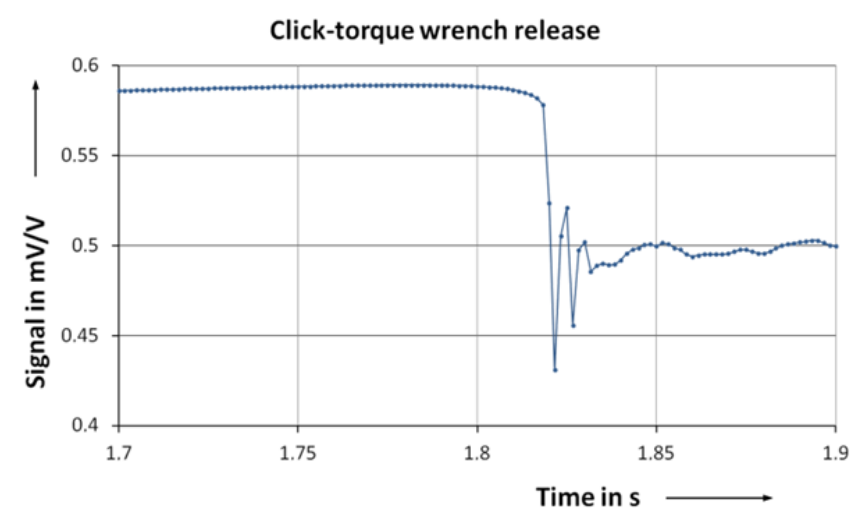

Figure 2. Torque signal of a releasing click-torque wrench over time.

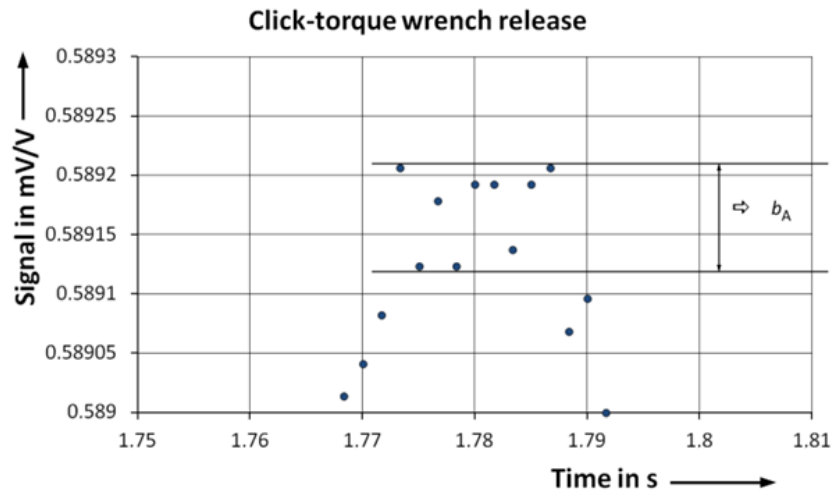

Figure 3. Determination of the release torque $A$ from the torque signal of a releasing click-torque wrench over time considering the contribution $b_{\mathrm{A}}$ of the signal noise to the measurement uncertainty.

Together with the relative uncertainties of the signal recording and of the sensitivity of the reference transducer, plus the influences of the reference transducer creeping and of the zero signal drift, the combined relative uncertainty of the determination of the release torque $A$ amounts to about $4 \cdot 10^{-4}$.

\subsection{Relative repeatability $s_{\text {rpt }}$}

As a mechanical mechanism, a CTW is affected by the history of the load, temperature and humidity. Every CTW in this investigation was stored under laboratory conditions for one day at least before use. Furthermore, preliminary series of releases were executed.

With this preliminary series of up to 120 releases, several purposes are to be served. First, the CTWs' lubrication after long idleness should be recovered and, second, the maximum release rate should be achieved. If releases succeed too fast, thermal effects in the release mechanism provoke a drift of the release torque value. Furthermore, the relative standard deviation of the values of $A$ provides the relative repeatability $s_{\mathrm{rpt}}$ of the mean value $\bar{A}$ of the release torque (Figure 4 ).

$S_{\mathrm{rpt}}=\sqrt{\frac{\sum_{i=1}^{n}\left(A_{i}-\bar{A}\right)^{2}}{n(n-1)}}$

Achieving the mean value of the release torque is a specific task of a transfer measurement. Usually measurements with a CTW according to ISO 6789 are never given as an average, because every single release in the calibration has to fulfil the demands of the standard.

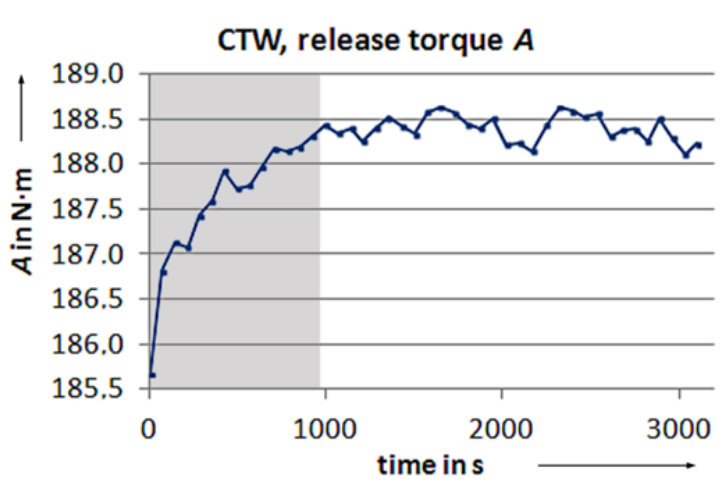

Figure 4. Devolution of the release torque $A$ of a CTW during consecutive releases. The running-in reaches to value No. 14 (shaded area) and the relative standard deviation of the rest of the data delivers a relative repeatability $s_{\text {rpt }}$ of $7.6 \cdot 10^{-4}$. 
Due to friction and resolution effects within the release mechanism, even without thermal effects the relative shortterm repeatability of consecutive releases can exceed $10^{-3}$. Series of 30 repeated release events at least were performed and averaged for the determination of each value of $\bar{A}$ in order to overcome this instability of the CTWs. This is important, as a high value of the repeatability relative to the other coefficients is to be taken into account at the uncertainty estimations of them.

\subsection{Relative reproducibility $s_{\mathrm{rpr}}$}

The relative reproducibility $s_{\text {rpr }}$ is defined as the relative difference between two independent measurements of $\bar{A}$ with one CTW.

Manufacturers of CTWs recommend readjusting them to a minimum value after each use to avoid mechanical drifting during long-term storage due to the spring tension inside the release mechanism. Nevertheless, keeping up the tension of the CTW over the days of investigation turned out to be advantageous. The drift that manufacturers warn against could not be detected during extensive tests of the specimens. Therefore, avoiding the readjustment of the CTWs between measurements yields the benefit of a smaller value of $s_{\text {rpr }}$ by eliminating the resolution uncertainty (Figure 5). The relative resolution of the CTW, which amounts from $2 \cdot 10^{-4}$ to $3 \cdot 10^{-3}$ for the specimens used in this work, is one of the main uncertainty contributions for calibrations according to ISO 6789. It originates from the uncertainty of the adjusting scale due to the width of lines and due to mechanical clearance in the adjusting mechanism, even if these are latching.

As usual, calibrations at the beginning and at the end of a transfer can deliver a reliable value of $s_{\mathrm{rpp}}$. In this work, these calibrations were simulated by repeated remounting of the CTW while the adjustment was kept.

\subsection{Relative coefficient of lever length $c_{1}$}

The distance between the pivot of the square drive and the support at the handhold of a CTW affects the amount of $A$ supposedly by a cross force effect in the pivot. Furthermore, deviations of $A$ correlative to the lever length could be caused by an eccentric female square drive in the calibration facility [2]. This has been avoided by careful alignment of the facility axis with an eccentricity of less than $0.05 \mathrm{~mm}$. So it is possible to detect just such an eccentricity in the calibration facility under test during a transfer calibration using the CTW, by gaining increased values of $c_{1}$ there.

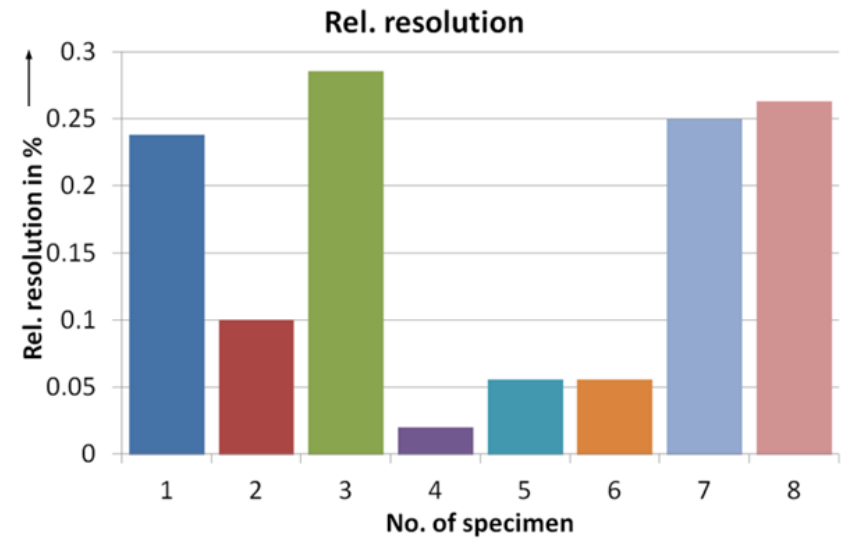

Figure 5. Amount of CTWs' relative resolution, given mainly by adjusting uncertainty.
To find the value of $c_{1}$, two additional series of 30 releases each are performed at a lever length $10 \mathrm{~mm}$ shorter respectively longer than the normal length. The relative coefficient of lever length thus can be found with

$c_{1}=\frac{S_{\text {long }}-S_{\text {short }}}{\bar{S}\left(l_{\text {long }}-l_{\text {short }}\right)}$

where $\bar{S}$ is the average of peak signals of the calibration facility gained at the preliminary series mentioned above, and $S$ is the averaged peak signal with the longer or the shorter lever respectively, which length is given by $l$.

Though the determination of $c_{1}$ usually has to be performed at minimum level of torque adjustment, for the purposes of transfer measurements this should be done preferably at nominal torque adjustment in this context. In this way, no readjustment of the CTW takes place and the uncertainty contribution of adjustment reproducibility can be avoided.

\subsection{Relative coefficient of temperature $c_{T}$}

The peak value of a CTW can be influenced by its temperature by way of altering the lubrication viscosity, by way of dimensional changes in the release mechanism or, if a strain gauge is integrated, by temperature dependence of the gauge sensitivity [3].

One possibility to get an estimation of $c_{\mathrm{T}}$ is to track the relaxation of a heated CTW from $40^{\circ} \mathrm{C}$ by repeated series of 30 release events each to the normal laboratory temperature of $21^{\circ} \mathrm{C}$. The CTW can be heated inside a climate cabinet overnight and then it will have to be moved quickly into the calibration facility, which is assisted by the quick and easy mounting of the CTW with a square drive. At the beginning of the experiment, the repetition rate of the series should be high. Because of the downtime of measurements during the transport of the CTW from the climate cabinet into the calibration facility, the initial status of the CTW is only available by an extrapolation. The relaxated state, in contrast, is to be measured simply by waiting some hours until the stability of occasional measurements is equal to the repeatability of the CTW obtained at the preliminary measurement. This method is equivalent to the method which was proposed for simplified measurements of the relative humidity coefficient [4].

With this set-up, $c_{\mathrm{T}}$ can be obtained by

$c_{\mathrm{T}}=\frac{S_{\mathrm{Cab}}-S_{\mathrm{Lab}}}{\bar{S}\left(T_{\mathrm{Cab}}-T_{\mathrm{Lab}}\right)}$

where $\bar{S}$ and $S$ are defined analogical to (2), but in the case of a temperature step $T_{\mathrm{Cab}}-T_{\mathrm{Lab}}$ between a climate cabinet and the laboratory.

In contrast to CTW calibration according to ISO 6789, transfer measurements can be reduced to very close limits of temperature, because these measurements are only reasonable in laboratories with low uncertainty budget and thereby with an efficient temperature control. Thus, the contribution of $c_{\mathrm{T}}$ in a transfer measurement usually remains small.

\subsection{Relative coefficient of humidity $c_{\mathrm{F}}$}

Like temperature, humidity can affect the lubrication of the mechanism or the sensitivity of a gauge within a CTW [5]. In a similar way as described above, the humidity of a CTW can be changed in a climate cabinet and its relaxation to the laboratory level can be observed. Then $c_{\mathrm{F}}$ is given by 
$c_{\mathrm{F}}=\frac{S_{\mathrm{Cab}}-S_{\mathrm{Lab}}}{\bar{S}\left(F_{\mathrm{Cab}}-F_{\mathrm{Lab}}\right)}$

with $\bar{S}$ and $S$ defined analogical to (2), but in the case of a humidity step $F_{\mathrm{Cab}}-F_{\mathrm{Lab}}$ between a climate cabinet and the laboratory.

Strict humidity control is challenging and expensive even for ambitious laboratories. Therefore, humidity deviation between PTB and a laboratory under test is usually greater than $5 \%$ rh, often greater than $10 \%$ rh. Consequently, the impact of $c_{\mathrm{F}}$ on the uncertainty budget potentially could be higher than that of other coefficients discussed in this paper.

\subsection{Relative coefficient of rise time $c_{\mathrm{tB}}$}

The rise time $t_{\mathrm{B}}$ of a CTW release event is defined as the time between $80 \%$ load and the release load which defines the $100 \%$ load. The dedicated relative coefficient $c_{\mathrm{t}}$ can be obtained by

$c_{\mathrm{tB}}=\frac{S_{\text {fast }}-S_{\text {slow }}}{\bar{S}\left(t_{\mathrm{B}, \text { fast }}-t_{\mathrm{B}, \text { slow }}\right)}$

with $\bar{S}$ and $S$ defined analogical to (2), but in the case of a step in the rise time $t_{\mathrm{B}, \text { fast }}-t_{\mathrm{B}, \mathrm{slow}}$.

Measurement of the rise time needs correction if the CTW under test exhibits a high reading error. Then the release torque is far away from the expected nominal value and the starting point of the rise time has to be shifted to $80 \%$ of the actual release torque. In the most cases, shifting to an earlier time is necessary, which is possible if the loading curve was recorded completely as given in this work. If a calibration facility delivers only the peak value of the curve, correction of the rise time value is impossible.

\subsection{Relative coefficient of square drive influence $c_{V}$}

The quality of the square drive can influence the result of a CTW calibration by reactive forces and moments which are possible if angularity, planarity and dimensional accuracy are inadequate. The usual procedure to determine $\propto$ is to perform five series of 10 measurements with orientation alterations of the square drive by $90^{\circ}$ between them.

During this investigation only a short version of this procedure was performed with 30 release events at $0^{\circ}$ and $90^{\circ}$ position of the square drive each, because of the great time need of such measurements. Then, a coefficient could be obtained using

$$
c_{\mathrm{V}}=\frac{S_{0^{\circ}}-S_{90^{\circ}}}{\bar{S}}
$$

with $\bar{S}$ and $S$ defined analogical to (2), but in the case of the alteration of the square drive position from $0^{\circ}$ to $90^{\circ}$.

Of course, transfer measurements should be performed with a fixed position of the square drive in both calibration facilities involved, but the determination of $c_{\mathrm{V}}$ does not become dispensable. Assuming the female square drive of the PTB facility to be well aligned, an increased value of $\omega_{\mathrm{V}}$ in the facility under test would indicate some of the mechanical problems described above at the female square drive of the latter. In this sense, $c_{\mathrm{V}}$ is due to the properties of the calibration facility in the first order. Therefore, the contribution of $c_{\mathrm{V}}$ to the measurement uncertainty of the CTW should be taken into account if $\propto$ is increased in the laboratory under test.

\subsection{Relative coefficient of the ratchet influence $c_{\text {rch }}$}

If the CTW comes with a ratchet, eccentricity of the rotating part of it is an important source of deviation, as in the case of eccentricity of the machine's axis, mentioned in section 2.6. about $c_{1}$.

Because the ratchet is not a part of the calibration facility under test, in a transfer measurement this influence should be eliminated by using a fixed or labelled position of the ratchet in both facilities. Since ratchets often come with high angular resolution and are not usually fixable, an alteration of the ratchet position could take place unnoticed during each handling. Thus, the effect of the ratchet position alteration was determined by

$c_{\mathrm{rch}}=\frac{S_{+1 \operatorname{cog}}-S_{-1 \operatorname{cog}}}{\bar{S}}$

with $\bar{S}$ and $S$ defined analogical to (2), but in the case of the alteration in the ratchet position by $\pm 1 \mathrm{cog}$. Because of the sinusoidal character of the eccentricity this measurement was undertaken at a ratchet position of $0^{\circ}$ and of $90^{\circ}$. The maximum value of $c_{\text {rch }}$ was used.

\subsection{Uncertainty of coefficients}

The uncertainties of the coefficient determinations described in this paragraph are dominated by that of $S$, which is given mainly by the repeatability $s_{\mathrm{rpt}}$ of the CTW. Therefore, the contribution of the coefficients to the uncertainty is at least in the range of $s_{\mathrm{rpt}}$ multiplied by a sensitivity coefficient given by the amount of alteration of the influence quantity in question (see section 4.4). This underlines the importance of small repeatabilities for the value of a CTW for transfer measurements. The use of at least 30 release events for the determination of each value of $\bar{A}$ reduces the influence of the release instability by more than a factor of 5 .

\section{SPECIMENS}

In order to obtain an overview of possible properties of CTWs, some different types of them were investigated in the manner described in the chapter above (Table 1).

Most of them are of the common mechanical release type. These wrenches are equipped with a release mechanism consisting of an instable crank, which can be pre-stressed by a spring and thereby be adjusted for a certain release torque. When the torque load exceeds the adjusted value, the instable crank turns over and the torque load at the square drive falls abruptly.

While this mechanism is integrated into the body of the mechanical CTW, a buckling CTW is constructed to fold

Table 1. CTWs used in this investigation.

\begin{tabular}{llll}
\hline No. & Type & $\begin{array}{l}\text { Nom. } \\
\text { torque in } \\
\mathrm{N} \cdot \mathrm{m}\end{array}$ & Release type \\
\hline 1 & M210 & 210 & mechanical \\
2 & S.305 DA & 350 & mechanical \\
3 & No 18 & 700 & buckling \\
4 & $714 / 10$ & 100 & electromechanical \\
5 & $5122 \mathrm{CT}$ & 180 & mechanical \\
6 & $1800 \mathrm{QL}$ & 180 & mechanical \\
7 & $721 \mathrm{Nf} / 80$ & 800 & mechanical \\
8 & Typ D & 760 & mechanical \\
\hline
\end{tabular}


entirely at the half-way point of the lever. This design is outdated and nowadays in use merely for screwing in workshops. One exponent of this type is used in the survey to have a look at the lower end of the state-of-the-art.

An electromechanical CTW includes a strain gauge to measure the torque load. When this measure exceeds a digitally preset limit, an electromagnetic force unlocks the square drive for release. This design is quite new, but nevertheless it raises expectations for solving some of the problems which are known about mechanical CTWs.

\section{RESULTS}

\subsection{Sampling rate}

To obtain a CTW with a well-defined and repeatable release value, the act of releasing has to be as short as possible.

The signal in time therefore tends to be discontinuous, which implies high requirements for the sampling rate of the amplifier which detects the release event. Comparisons of release measurements with different sampling rates exhibit relative deviations of the peak value up to $1.3 \cdot 10^{-3}$ (Figure 6).

Using a signal of a release event measured with a sampling rate of $9.6 \mathrm{kHz}$, a simulation of signal integrations with time periods corresponding to lower sampling rates was calculated. This simulation curve shows a qualitative shape similar to that of the measurements. According to that, the curve should change from a high inclination at lower sampling rates to an approximately constant part for rates higher than $2 \mathrm{kHz}$. Therefore it is advisable for a comparison of two calibration facilities either to use a sampling rate of more than $2 \mathrm{kHz}$ or to agree on a certain value of the sampling rate, if only lower rates are available.

\subsection{Rise time}

In daily workshop use a CTW is loaded with torque by hand and as quickly as the worker is able to. Thus, the typical rise time of torque is shorter than one second. To accommodate this fact, in ISO 6789 values of $t_{\mathrm{B}}$ are required to be within $0.5 \mathrm{~s}$ and $4 \mathrm{~s}$. The higher value is far beyond the practical value for manual loading and is to be seen as a concession to the deficient speed of calibration machines.

The mechanical parts of CTWs are subject to inertia effects and to friction. Both depend on the velocity of the moving parts. In this way, rise time can affect the release torque value in CTWs. Electromechanical CTWs additionally have to face the runtime error of the release bolt and the influences of the sensing element resolution and of the internal sampling rate. In
Influence of sampling rate on peak value

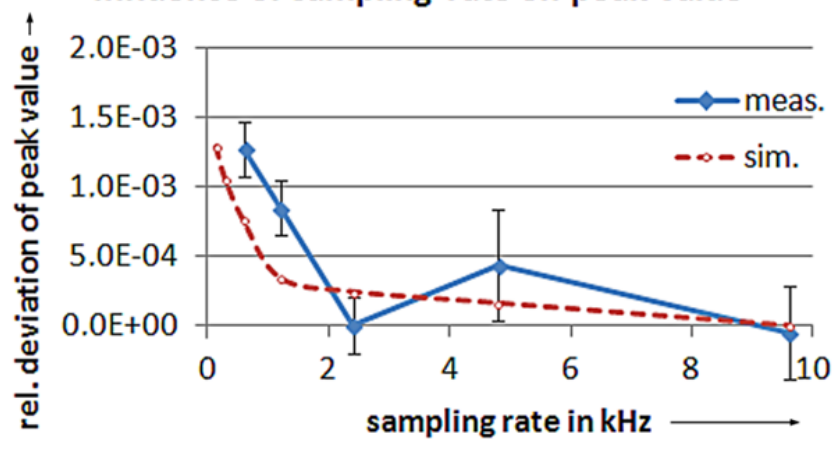

Figure 6. Relative deviation of peak value, depending on the sampling rate of the amplifier. The dashed curve is the result of a simulation which performs signal integrations with corresponding time periods at a measured release signal gained at a sampling rate of $9.6 \mathrm{kHz}$.

this spirit, a rise time of $0.5 \mathrm{~s}$ is unreasonably short for transfer measurements.

In this connection, the limits of ISO 6789 are too broad for practical application and too close for the requirements of transfer measurements. The situation becomes worse if the calibration facility under test lacks a precise rise time gauging. Then the transfer measurement not only has to test the release torque measurement capability of the calibration machine under test. Besides this, the procedure should provide information about the rise time of the calibration machine in question.

Measurements with different rise times show a relative deviation of the release torque of some $10^{-3}$ per second at rise times shorter than $4 \mathrm{~s}$, in agreement with the consideration above (Figure 7).

In the range between $4 \mathrm{~s}$ and $5 \mathrm{~s}$ the dependence of the release torque on the rise time is minimal. Thus, for the purpose of transfer, measurements should be performed slowly with a rise time of $5 \mathrm{~s}$.

As manufacturers are obliged to design their calibration machines according to ISO 6789, the machines are often unable to run so slowly. In this case, the two comparing laboratories should agree upon rise time, which poses the problem of measuring the rise time exactly.

\subsection{Selection of a transfer CTW}

In order to rate the ability of the CTWs for transfer purposes, a table of the coefficients of the CTWs in the survey is given (Table 2).

Table 2. Absolute values of relative coefficients in $10^{-6}$ of CTWs listed in Table 1 . Some data were not measured due to time constraints; the referring coefficients were treated as equalling zero. The deviation of specimen No. 3 is due to friction-induced drift and was treated as a systematic contribution.

\begin{tabular}{|c|c|c|c|c|c|c|c|c|}
\hline $\begin{array}{l}\text { rel. coefficient } \\
\text { in } 10^{-6}\end{array}$ & 1 & 2 & 3 & 4 & 5 & 6 & 7 & 8 \\
\hline$c_{1}$ in $\mathrm{mm}^{-1}$ & 2548 & 92 & no data & 326 & 394 & 636 & 330 & 37 \\
\hline$c_{\mathrm{T}}$ in $\mathrm{K}^{-1}$ & 1041 & 351 & no data & 507 & 903 & 510 & 1008 & 181 \\
\hline$c_{F}$ in $(\% \mathrm{rh})^{-1}$ & 1154 & 560 & no data & 20 & 268 & 77 & no data & 229 \\
\hline$c_{\mathrm{tB}}$ in $\mathrm{s}^{-1}$ & 8153 & 6029 & no data & 2293 & 5749 & 1259 & 4537 & 3084 \\
\hline$c_{\mathrm{V}}$ & no data & 4071 & no data & 220 & 7453 & 586 & 151 & 8041 \\
\hline$c_{\text {rch }}$ (one cog) & 6787 & 1249 & no data & 305 & 5783 & 2316 & 2635 & no ratchet \\
\hline$S_{\mathrm{rpr}}$ & 2748 & 217 & no data & 143 & 3419 & 674 & 989 & 5184 \\
\hline$s_{\mathrm{rpt}}$ & 580 & 234 & 27842 & 96 & 139 & 524 & 646 & 334 \\
\hline
\end{tabular}


Influence of rise time on peak value

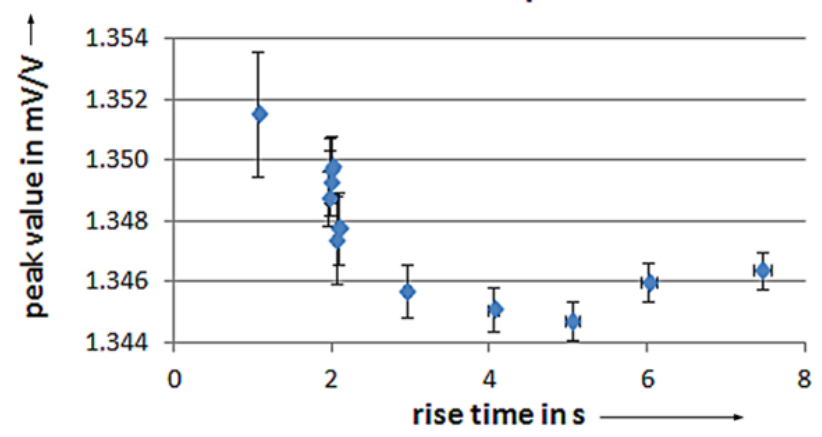

Figure 7. Peak value of a CTW depending on the torque load rise time.

This survey is neither representative of the CTWs available on the market nor could a conclusion be drawn about the properties of a CTW type, this would require tests at a higher number of CTWs of a certain type. The survey shall give examples of which parameters are important. Better than a competition of coefficients, an analysis of their uncertainty impacts give evidence of the suitability of a CTW for transfer calibrations (Table 3, Figure 8).

The contributions in Table 3 indicate the priority for

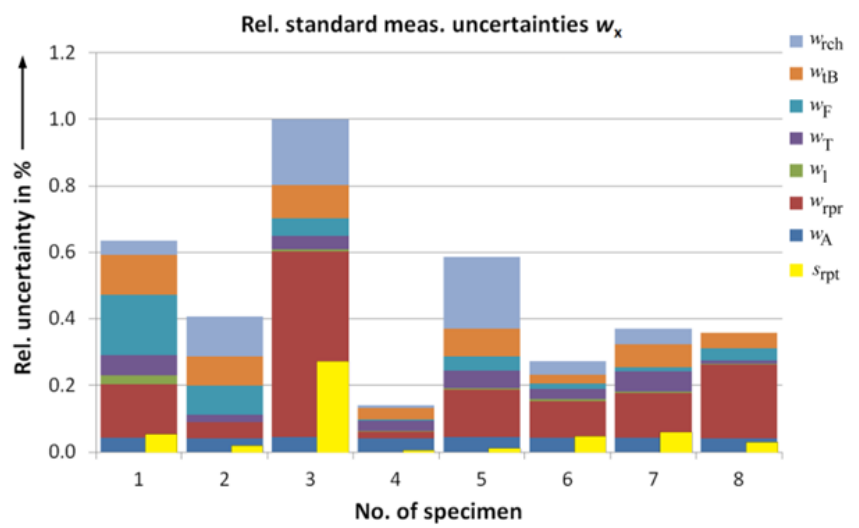

Figure 8 . Relative standard uncertainties of the investigated contributions calculated according to (9), (10) and (11). compensating the uncertainty sources of a CTW or for its improvement in order to qualify it for transfer measurements. The most influential contribution to the measurement uncertainty to be considered at the selection of a transfer CTW is the repeatability $s_{\mathrm{rpt}}$.

Further parameters not included in Table 2 are the object of a qualitative appraisal. The shape of a release curve should feature a well-defined peak value with a monotone rising and a fast break off, kickback-free for at least one second. The specific running-in of the release value from the initial increase to stable amounts has to be observed at preliminary tests and is to be considered at the calibrations by omitting the referring data points. Moreover, it has to be ensured by tests that keeping the CTW in tension during a transfer calibration would not increase repeatability uncertainty due to mechanical drift as mentioned in section 2.5 .

\subsection{Uncertainty budgets}

The combined relative uncertainty of the reference torque measurement with a CTW $w_{\text {Ref }}$ consists of the contributions of the reproducibility $w_{\mathrm{rpr}}(9)$, of the release torque detection $w_{\mathrm{A}}$ (10) and of the influence of the coefficients $w_{\mathrm{x}}(11)$ :

$w_{\mathrm{Ref}}=\sqrt{w_{\mathrm{rpr}}^{2}+w_{\mathrm{A}}^{2}+\sum_{x} w_{\mathrm{x}}^{2}}$

$w_{\mathrm{rpr}}=\sqrt{\frac{s_{\mathrm{rpr}}^{2}}{12}+2 s_{\mathrm{rpt}}^{2}}$

$w_{\mathrm{A}}=\sqrt{2 s_{\mathrm{zero}}^{2}+w_{\mathrm{NN}}^{2}+s_{\mathrm{NN}}^{2}+\frac{b_{\mathrm{A}}^{2}}{12}}$

The contribution of the reproducibility is given by the measured amount of $s_{\mathrm{rpr}}$ and twice the uncertainty of this measurement which is dominated by the repeatability $s_{\mathrm{rpt}}$.

The contribution $w_{\mathrm{A}}$ consists - in addition to the span $b_{\mathrm{A}}$ of the relative uncertainty of the national standard facility $w_{\mathrm{NN}}$, the relative long-term stability of the facility $s_{\mathrm{NN}}$ and twice the relative stability of the zero signal of the standard facility.

Table 3. Relative standard measurement uncertainties $w$ in \% of CTWs listed in Table 1 due to examined quantities introduced in the text for a transfer measurement between a national standard machine and a calibration laboratory. The contributions of the specific coefficients listed in Table 2 are calculated using the calibration conditions listed in Table 4 both for a standard machine and for a calibration laboratory. The resulting combined expanded relative uncertainty $W_{\text {Ref }}$ is given both under the assumptions given in Table 4 and neglecting the influence of a ratchet. The contributions, except for $w_{\text {rch, }}$ are graded in reference to their amount by colour. The highest contributions are printed in red, the second highest in orange.

\begin{tabular}{|c|c|c|c|c|c|c|c|c|}
\hline uncertainty in \% & 1 & 2 & 3 & 4 & 5 & 6 & 7 & 8 \\
\hline$w_{\mathrm{A}}$ & 0.042 & 0.041 & 0.044 & 0.041 & 0.044 & 0.043 & 0.042 & 0.041 \\
\hline$W_{\text {rpr }}$ & 0.161 & 0.048 & 0.557 & 0.020 & 0.142 & 0.108 & 0.135 & 0.222 \\
\hline$w_{1}$ & 0.026 & 0.001 & 0.007 & 0.003 & 0.004 & 0.007 & 0.004 & 0.001 \\
\hline$W_{\top}$ & 0.063 & 0.021 & 0.041 & 0.030 & 0.054 & 0.031 & 0.061 & 0.012 \\
\hline$w_{\mathrm{F}}$ & 0.180 & 0.087 & 0.053 & 0.004 & 0.042 & 0.016 & 0.012 & 0.036 \\
\hline$W_{\text {rch }}$ & 0.041 & 0.119 & 0.197 & 0.009 & 0.215 & 0.041 & 0.046 & no ratchet \\
\hline$W_{\text {Ref }}, k=2$ & 0.57 & 0.37 & 1.29 & 0.13 & 0.57 & 0.26 & 0.35 & 0.47 \\
\hline $\begin{array}{l}W_{\text {Ref, }} k=2 \\
\text { (no ratchet) }\end{array}$ & 0.56 & 0.28 & 1.12 & 0.13 & 0.37 & 0.25 & 0.34 & 0.47 \\
\hline
\end{tabular}


Table 4. Uncertainty of calibration conditions $u_{x}$ expressed as a half-span value used for the calculation of an uncertainty budget in a transfer measurement (Table 3). Given are measured values of the PTB standard calibration machine (NN) and assumed values for typical accredited calibration laboratories (Lab).

\begin{tabular}{l|c|l|l} 
Condition & Index $x$ & $u_{\mathrm{x}}(\mathrm{NN})$ & $u_{\mathrm{x}}$ (Lab) \\
\hline Lever length & $\mathrm{I}$ & $0.25 \mathrm{~mm}$ & $0.25 \mathrm{~mm}$ \\
Temperature & $\mathrm{T}$ & $0.5 \mathrm{~K}$ & $2 \mathrm{~K}$ \\
Humidity & $\mathrm{F}$ & $2 \% \mathrm{rh}$ & $5 \% \mathrm{rh}$ \\
Rise time & $\mathrm{tB}$ & $0.1 \mathrm{~s}$ & $0.5 \mathrm{~s}$ \\
Ratchet position & $\mathrm{rch}$ & $0 \mathrm{cog}$ & $1 \mathrm{cog}$
\end{tabular}

The coefficients $c_{\mathrm{x}}$ (x stands for one of the indices $1, \mathrm{~T}, \mathrm{~F}$, $\mathrm{tB}$ and rch introduced in chapter 2$)$, stability contributions $s_{\mathrm{rpr}}$ and $s_{\text {rpt }}$, uncertainty of calibration conditions $u_{\mathrm{x}}$ (Table 4), and distribution functions were processed to relative standard uncertainty contributions $w_{\mathrm{x}}$ under the condition that the sampling rate is chosen adequately and $c_{\mathrm{v}}$ is equal in both laboratories. Because the complete analysis according to the Guide to the Expression of Uncertainty in Measurement [6] is disproportionately extensive, a simplified approximation was used:

$w_{x}=\left(u_{x}(\mathrm{NN})+u_{\mathrm{x}}(\mathrm{Lab})\right) \sqrt{\frac{c_{\mathrm{x}}^{2}}{12}+2\left[\frac{S_{\mathrm{rpt}}}{\Delta x}\right]^{2}}$

While the uncertainty of the coefficient's detection - given mainly by the repeatability $s_{\mathrm{rpt}}$ - cannot be neglected, the contribution of a specific coefficient $c_{\mathrm{x}}$ has to be extended by the $\Delta x^{\text {th }}$ fraction of $s_{\text {rpt. }}$. Here, $\Delta x$ is the step of the calibration condition quantity, in units of this quantity, performed during the detection of the coefficient. The contribution of the coefficient $c_{\mathrm{x}}$ is derived from a span, thus a rectangular distribution is to be taken into account. In contrast, the repeatability $s_{\text {rpt }}$ originates from an averaging, thus a normal distribution is to be assumed. Because $c_{\mathrm{x}}$ is achieved by a difference, the extension due to the repeatability is to be taken into account twice.

To obtain the actual contribution of the coefficients, the expanded specific coefficients have to be multiplied by the uncertainty of the corresponding calibration conditions $u_{\mathrm{x}}$, which are given in Table 4 for the reference calibration with the national standard machine (NN) and for a typical accredited calibration laboratory (Lab). Thus the relative standard uncertainty $w_{\mathrm{x}}$ includes the impact of the CTW properties at both calibration facilities involved.

The uncertainty contribution of the stability $w_{\text {rpr }}$ is calculated equivalent to the second part of (11) from the reproducibility $s_{\mathrm{rpr}}$ with a rectangular distribution and twice the repeatability $s_{\mathrm{rpt}}$ with a normal distribution.

As an overall result, the expanded relative combined uncertainty $W_{\text {Ref }}(k=2)$ is given for each CTW in Figure 9. This uncertainty implies the contributions due to CTW properties in combination both with the calibration conditions in the national standard machine (TN-NN) and with those in a calibration machine of a laboratory under test (TN-Lab) as well as a contribution from the national standard machine (NN). If a transfer calibration should confirm the bmc of a calibration machine under test, the $E_{\mathrm{n}}$ value (12) must not exceed 1:

$\left|E_{\mathrm{n}}\right|=\left|\frac{\bar{A}_{\mathrm{NN}}-\bar{A}_{\mathrm{Lab}}}{\sqrt{U_{\mathrm{Ref}}^{2}+U_{\mathrm{Lab}}^{2}}}\right| \leq 1$
The $E_{\mathrm{n}}$ value compares the difference between two measurements of the release torque $\bar{A}$ with the quadratic sum of uncertainties referring to these calibrations. In this work, the transfer CTW is to be understood as a part of the reference calibration, thus the referring expanded uncertainty $U_{\text {Ref }}$ corresponds to the relative expanded uncertainty $W_{\text {Ref given in }}$ Figure 9:

$U_{\mathrm{Ref}}^{2}=U_{\mathrm{NN}}^{2}+U_{\mathrm{TN}-\mathrm{NN}}^{2}+U_{\mathrm{TN}-\mathrm{Lab}}^{2}$

The examination of $U_{\text {Ref }}$ is important since (12) implies that even at equality of the two comparison measurements $\left(\bar{A}_{\mathrm{NN}}=\bar{A}_{\mathrm{Lab}}\right)$, the smallest bmc the comparison could verify is $U_{\text {Lab }}=U_{\text {Ref. }}$. Therefore, a CTW for transfer application without restriction in accredited laboratories according to Figure 1 should feature a value of $W_{\text {Ref }}$ smaller than $0.2 \%$. Only specimen No. 4 meets this requirement and is able to be used in transfer measurements for all laboratories. The majority of bmc values in Figure 1 is greater than or equal to $0.5 \%$. Transfer to these laboratories should be possible also with CTW No. 6 with a sufficient uncertainty buffer.

The best results were achieved with CTW No. 4 which is of the electromechanical type. Apparently the higher effort in engineering is reflected in a very low dependency of the release torque on calibration conditions.

In Table 3, the contributions are graded in reference to their amount by colour. The highest contributions are printed in red, the second highest in orange. Excluded is $w_{\text {rch }}$, because this contribution could be avoided by using no ratchet but rather a fixed square drive.

For CTW No. 4, the contribution of $w_{\mathrm{A}}$, which is mainly the uncertainty of the standard calibration facility, is the highest, the contribution of $w_{\mathrm{tB}}$ is the second highest. A further improvement of this CTW should start with an enhancement of the internal sampling rate, which should reduce the influence of the rise time. For the other CTWs, an improvement of this aspect is only to be expected by re-designing the releasing parts - quite a large effort which does not meet the actual goals of the manufacturers. But many of the CTWs could be improved fundamentally in this field.

Most of the CTWs would benefit also from an improvement of the relative repeatability $s_{\mathrm{rpt}}$. This is indicated in Table 2 by the amount of the repeatability of a CTW in comparison to the other influences. $s_{\mathrm{rpt}}$ should be in the range

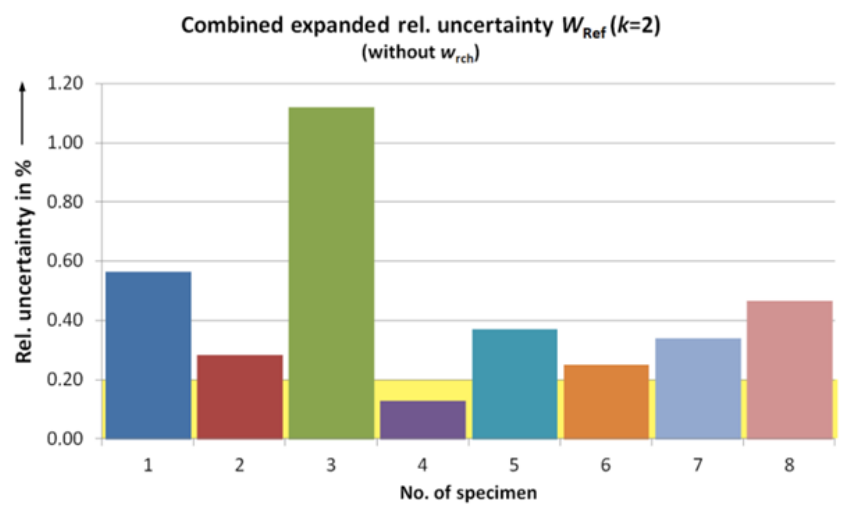

Figure 9. Combined expanded relative uncertainty $W_{\text {Ref }}$ for the investigated specimens in comparison to the smallest bmc of the DAkkS-accredited laboratories (yellow bar). 
of the smallest contribution in this compilation. Many of the tested CTWs cannot fulfil this requirement.

CTW No. 3 exhibits friction-induced drift during the preliminary measurements. This drift is to be understood as a systematic contribution and therefore has to be taken into account by absolute value. Thus, this contribution on its own exceeds the requirements of transfer application and hence no more measurements were performed with this CTW. Coefficients which were not measured are marked in Table 2 with "no data". These are counted as zero in order to get a lower estimation of $W_{\text {ref }}$ at the end. Nevertheless, the uncertainty contributions $w_{\mathrm{x}}$ of these conditions are greater than zero in this analysis because of the contribution of $s_{\mathrm{rpt}}$ in (11).

\subsection{Procedure for transfer measurements}

The measurements of the survey show that the procedure of ISO 6789 (Figure 10) is not adequate for transfer measurements with CTWs.

Readjusting uncertainty of the CTWs can be avoided when the adjustment of the CTW is not changed during the measurements. This adjustment should be made at the nominal value of the CTW in order to use the best possible signal-tonoise ratio. Extensive tests with CTWs stored several days under the tension of this adjustment did not show a drift of the release torque. Furthermore, the CTW with the best overall uncertainty is of the electromechanical type and hence is free of mechanical tension due to adjustment.

The transfer measurement should consist of series of at least 30 release events to reduce the repeatability uncertainty (Figure 11). The load rise time should be fixed to a certain value, preferably at $5 \mathrm{~s}$. Furthermore, the laboratories should agree on a fixed value of the sampling rate, at best more than $2 \mathrm{kHz}$. The position of the square drive and of the ratchet should be the same in both laboratories. Additional series after alteration of the square drive position by $90^{\circ}$ should be performed at least for two positions. The length of the lever, the temperature and the humidity should be controlled and documented.

\section{CONCLUSIONS}

The capability of CTWs for use as transfer transducers depends not only on their technical specifications. Restricted specifications could be compensated if adapted procedures are employed which can largely differ from those according to ISO 6789. The objective of these procedures should be an

ISO 6789 schedule

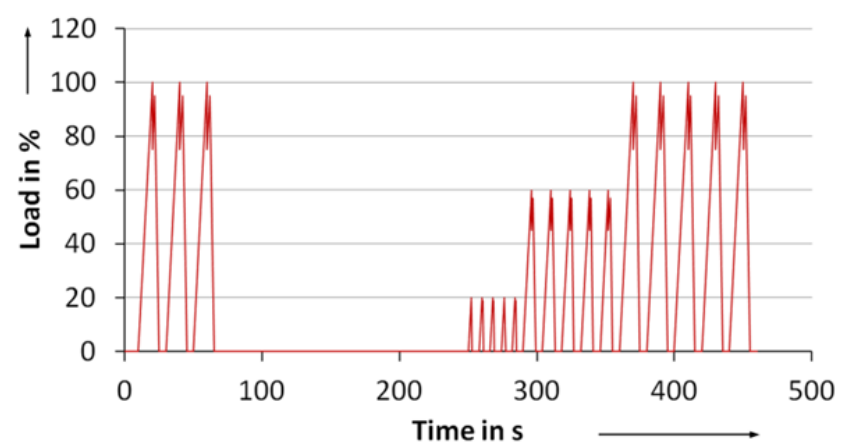

Figure 10. Loading schedule according to ISO 6789. optimization of the reproducibility of the CTWs' response. A suggested procedure designates the measurements required in ISO 6789 at $20 \%$ and $60 \%$ of the nominal value, but calls for at least 30 repeated measurements instead of 5. Parameters like rise time, positions of square drive and ratchet, sample rate, temperature, humidity and lever length are to be agreed upon and reproduced in the laboratories within narrow limits.

The survey delivers the following results:

1. The use of transfer CTWs for DAkkS assessments is possible under special conditions and with adequate types of CTWs for the laboratories accredited by the DAkkS. Therefore, the development both of specific measurement procedures and of selection criteria for CTWs, which was carried out to complement the traceability of the laboratories working in the field of ISO 6789, should be supplemented by further improvement of the CTWs, especially of their repeatability and rise time sensitivity.

2. The detected coefficients of the used specimens vary over a wide range of amounts. The selection of a CTW for transfer application therefore requires the analysis of the combined measurement uncertainty budget in the setup of the intended transfer measurement.

3. A new design of CTW with an electromechanical release mechanism yields the best results among the tested specimens. Here further improvements could be possible with higher internal sampling rates.

\section{ACKNOWLEDGMENT}

Thanks go to Sebastian Kaletka, who carefully performed hundreds of measurements for this investigation.

\section{REFERENCES}

[1] ISO 6789:2003-10, “Assembly tools for screws and nuts - Hand torque tools - Requirements and test methods for design conformance testing, quality conformance testing and recalibration procedure", International Organization for Standardization, Geneva, Switzerland.

[2] Brüge A., "Influence of eccentric mounting on the calibration of torque measuring devices", Proceedings of the 15th IMEKO TC3 Conference, October 7-11, 1996, Madrid, Spain, pp. 255259.

[3] Sanponpute, T.; Arksonnarong N., "Temperature and humidity dependence on stability of torque measuring devices", IMEKO $22^{\text {nd }}$ TC3, $15^{\text {th }}$ TC5 and $3^{\text {td }}$ TC22 International Conferences, February 3-5, 2014, Cape Town, Republic of South Africa

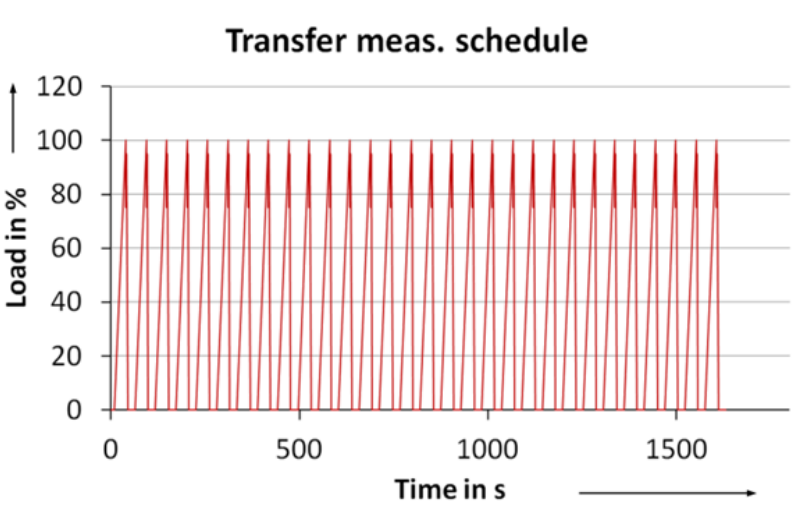

Figure 11. Proposed loading schedule for transfer measurement with CTW. 
URL: http://www.imeko.org/publications/tc3-2014/IMEKOTC3-2014-006.pdf

[4] Brüge A., "Influence of humidity on torque transducers Estimation methods for calibration laboratories", XX IMEKO World Congress, Metrology for Green Growth, 9 - 14 Sept. 2012, Busan, Republic of Korea.

URL:http://www.imeko.org/publications/wc-012/IMEKO-

WC-2012-TC3-O30.pdf
[5] Röske, D.; Mauersberger, D., "On the stability of measuring devices for torque key comparisons", IMEKO XVIII World Congress and IV Brazilian Congress of Metrology, "Metrology for a Sustainable Development", Rio de Janeiro, 17-22, September, 2006, Brazil, on CD-ROM, file name: 00181.pdf

[6] BIPM, "Evaluation of measurement data - Guide to the expression of uncertainty in measurement", JCGM 100:2008 URL:http://www.bipm.org/utils/common/documents/jcgm/J CGM_100_2008_E.pdf 